\title{
Liberalisierung und Privatisierung öffentlicher Dienstleistungen und die Erosion des Flächentarifvertrags
}

\author{
Torsten Brandt \\ Thorsten Schulten
}

\begin{abstract}
Mit der Liberalisierung und Privatisierung öffentlicher Dienstleistungen sind weitreichende Konsequenzen für das deutsche Tarifvertragssystem verbunden. Mit der Schaffung neuer Märkte im Bereich der öffentlichen Daseinsvorsorge werden zahlreiche Branchen von dem vormals umfassenden Tarifwerk des öffentlichen Dienstes abgekoppelt. Dabei entstehen jedoch keine neuen Branchentarifverträge. Es kommt vielmehr zur Herausbildung hochgradig fragmentierter Tarifvertragssysteme, die kaum in der Lage sind, die Konkurrenz um Lohn- und Arbeitskosten zu begrenzen. Die Liberalisierung und Privatisierung fördert damit die weitere Erosion des Flächentarifvertrags. ${ }^{1}$
\end{abstract}

\section{Einleitung}

Unter der Überschrift „Krise“ oder „Erosion“ des Flächentarifvertrags hat seit dem Ende der 1990er Jahre eine Vielzahl von Studien den grundlegenden Wandel des deutschen Tarifvertragssystems analysiert. ${ }^{2}$ Im Mittelpunkt dieser Untersuchungen stehen vor allem der anhaltende Rückgang der Tarifbindung (Ellgut/Kohaut 2008) sowie die zunehmende Tendenz zur Dezentralisierung und Verbetrieblichung der Tarifpolitik, die in der wachsenden Anzahl von Haustarifverträgen und der zunehmenden Bedeutung von Öffnungsklauseln zum Ausdruck kommen. Letztere erlauben Unternehmen, unter bestimmten Bedingungen vom Flächentarifvertrag nach unten abzuweichen (Bispinck 2006a; Kohaut/ Schnabel 2007).

In den Debatten über die Ursachen für diese Entwicklung dominierte über lange Zeit vor allem die "Globalisierungsthese“: Demnach sei der im nationalen Rahmen verhaftete Flächentarifvertrag angesichts der zunehmenden Internationalisierung der Ökonomie nicht mehr in der Lage, seine klassische Kartellfunktion wahrzunehmen und einheitliche Wettbewerbsbedingungen für eine bestimmte Branche zu definieren. Als besonders deutlicher Beleg für diese These gelten insbesondere die zahlreichen „betrieblichen Bündnisse für Arbeit", in denen mit Verweis auf die internationale Standortkonkurrenz Abweichungen vom Flächentarifvertrag vereinbart wurden (Rehder 2003).
Bei der „Globalisierungsthese “ wird die Krise des Flächentarifvertrags vor allem in den Industriebranchen verortet. Dies ist insofern erstaunlich, weil gerade in diesen Sektoren trotz aller Veränderungen nach wie vor eine relativ hohe Tarifbindung und relativ stabile Tarifvertragsstrukturen bestehen. Demgegenüber sind es vor allem die eher binnenwirtschaftlich orientierten Branchen des Dienstleistungssektors, in denen die Tariferosion vergleichsweise weit fortgeschritten ist (Ellgut/Kohaut 2008). Sie sind erst mit der Diskussion über den sich ausbreitenden Niedriglohnsektor in Deutschland stärker ins Blickfeld geraten (Bispinck 2007; Bispinck/Schulten 2008). Offensichtlich ist es hier weniger die Globalisierung als das Fehlen gewerkschaftlicher Organisations- und Durchsetzungsmacht und die arbeitsmarktpolitische Förderung prekärer Beschäftigungsverhältnisse, die die Erosion der Tarifvertragsstrukturen begünstigt haben.

In den Diskussionen um die Krise des Flächentarifvertrags völlig unterbelichtet sind schließlich die Veränderungen, die innerhalb des öffentlichen Sektors stattgefunden haben. Aufgrund seiner nach wie vor nahezu flächendeckenden Tarifbindung wird der öffentliche Dienst immer noch als weitgehend stabiles tarifpolitisches Terrain angesehen (Schnabel 2007). Dabei werden nicht nur die internen Veränderungen und Differenzierungen in den Tarifbeziehungen des öffentlichen Dienstes ignoriert (Dribbusch/Schulten 2007; Keller 2007). Es wird vor allem übersehen, dass im Zuge der Liberalisierung und Privatisierung öffentlicher Dienstleistungen zahlreiche Branchen vom Tarifvertragssystem des öffentlichen Dienstes entkoppelt wurden und neue - oftmals hochgradig fragmentierte - Tarifvertragsstrukturen entstanden sind. Diese Veränderungen sind für die Entwicklung des deutschen Tarifvertragssystems jedoch von mindestens genau so großer Bedeutung wie die Dezentralisierung im Industriesektor oder die Erosion der Tarifvertragsstrukturen in den privaten Dienstleistungsbranchen.

\footnotetext{
1 Der Artikel basiert auf Forschungsarbeiten, die im Rahmen des von der Europäischen Kommission geförderten internationalen Forschungsprojektes „Privatisation of Public Services and the Impact on Quality, Employment and Productivity" (PIQUE) (Projekt Nummer: CIT5-2006-028478) durchgeführt wurden, an dem das Wirtschafts- und Sozialwissenschaftliche Institut (WSI) in der HansBöckler-Stiftung als deutscher Projektpartner beteiligt ist. Für weitere Informationen siehe: www.pique.at.

2 Vgl. u. a. Artus (2001); Bispinck (2006a, 2007); Bispinck/Schulten (2003); Oppolzer/Zachert (2000); Kohaut/Schnabel (2003, 2007).
}

Torsten Brandt, Wissenschaftler im WSI in der Hans-Böckler-Stiftung (Projekt PIQUE). Arbeitsschwerpunkte: Privatisierung öffentlicher Dienstleistungen, Arbeitsmarktpolitik, Zukunft der sozialen Sicherung. e-mail: Torsten-Brandt@boeckler.de Thorsten Schulten, Dr., Wissenschaftler im WSI in der Hans-Böckler-Stiftung. Arbeitsschwerpunkte: Arbeits- und Tarifpolitik in Europa.

e-mail: Thorsten-Schulten@boeckler.de 


\section{Das Tarifvertragssystem des öffentlichen Dienstes im Umbruch}

Der öffentliche Dienst bildete traditionell einen der zentralen Stützpfeiler des deutschen Tarifvertragssystems. Mit dem Bundesangestelltentarifvertrag (BAT) und den entsprechenden Tarifverträgen für die Arbeiter (MTArb und BMT) existierte bis vor wenigen Jahren ein zentrales Tarifwerk, dessen Reichweite von keinem anderen Flächentarifvertrag übertroffen wurde (Keller 1993). De facto handelte es sich hierbei um Multibranchentarifverträge, die neben den Verwaltungen von Bund, Ländern und Gemeinden auch weite Bereiche der öffentlichen Daseinsvorsorge umfassten. Hinzu kamen weitere Sektoren, für die die Tarifabschlüsse im öffentlichen Dienst die zentrale Orientierungsmarke bildeten, die sie weitgehend übernahmen. Hierzu gehörten die tarifvertraglich schon immer eigenständig organisierten Branchen Bahn und Post sowie der gesamte Gesundheits- und Sozialsektor mit den großen Wohlfahrtsverbänden. Schließlich wurden mit den Tarifabschlüssen auch die Standards für die gesetzlich regulierte Beamtenbesoldung gesetzt.

$\mathrm{Da}$ die Tarifverträge für den gesamten öffentlichen Dienst in zentralisierten Verhandlungen auf nationaler Ebene vereinbart wurden, wurden überall weitgehend einheitliche Lohn- und Arbeitsbedingungen festgelegt. Das spezifische Arbeitsregime des öffentlichen Dienstes unterschied sich dabei von dem privaten Sektor vor allem durch ein deutlich höheres Maß an Beschäftigungssicherheit sowie eine deutlich geringere Lohnspreizung zwischen den einzelnen Beschäftigtengruppen (Atzmüller/Hermann 2004a). Während hoch qualifizierte Beschäftigte im öffentlichen Dienst eher weniger als in der Privatwirtschaft verdienten, war es bei weniger qualifiziertem Personal meistens umgekehrt.

Seit den frühen 1990er Jahren befindet sich der öffentliche Sektor jedoch in einem weitreichenden Restrukturierungsprozess, der nicht ohne Rückwirkungen auf das Tarifvertragssystem blieb (Dribbusch/Schulten 2007). Unter dem fiskalpolitischen Druck knapper Haushaltskassen wurden mit der Einführung von „New Public Management"-Konzepten zunehmend pri- vatwirtschaftliche Steuerungsmethoden eingeführt, die die als ,ineffizient" kritisierte öffentliche Verwaltung modernisieren sollten (Bordogna 2007). Hinzu kamen die zunehmende Ausgliederung und Privatisierung öffentlicher Dienstleitungen sowie die durch die Liberalisierung forcierte Konkurrenz von öffentlichen Unternehmen mit neu entstandenen privaten Dienstleistern.

Als ein Ergebnis dieser umfassenden Restrukturierungsphase kam es zu einem massiven Abbau öffentlicher Beschäftigung. So ist die Anzahl der Beschäftigten im öffentlichen Dienst zwischen 1991 und 2006 um mehr als zwei Millionen zurückgegangen. Etwa die Hälfte des Beschäftigungsrückgangs kann hierbei auf Privatisierungen zurückgeführt werden, während die andere Hälfte das Resultat von Rationalisierungsmaßnahmen im verbleibenden öffentlichen Dienst ist (Brandt/Schulten 2008a, S. 70f.). Der zunehmende Kostenund Effizienzdruck bildete darüber hinaus auch den Hintergrund, vor dem eine grundlegende Reform der Tarifverträge des öffentlichen Dienstes verhandelt wurde, die schließlich 2005 in die Verabschiedung des neuen Tarifvertrags Öffentlicher Dienst (TV-ÖD) mündete (Keller 2007). Dieser enthält eine Reihe von Öffnungsklauseln, die eine weitere Dezentralisierung und Ausdifferenzierung von Tarifverhandlungen ermöglichen, was insbesondere im Hinblick auf die Gestaltung der Arbeitszeiten in den folgenden Tarifrunden auch angewendet wurde (Bispinck 2006b). Bereits während der Verhandlungen über den neuen TV-ÖD kam es 2003 auf Arbeitgeberseite zum Bruch der Tarifgemeinschaft von Bund, Ländern und Kommunen, in dessen Folge die Länder seit 2006 auf Basis des Tarifvertrags für den öffentlichen Dienst der Länder (TV-L) eigenständige Tarifverhandlungen führen.

Insgesamt haben bereits die internen Veränderungen im Tarifvertragssystem des öffentlichen Dienstes zu einer erheblichen Differenzierung von Tarifstandards geführt, die die Ordnungsfunktionen des Flächentarifvertrags unterminieren. Hinzu kommen nun die durch Liberalisierung und Privatisierung ausgelösten externen Veränderungen, die zur (teilweisen) Entkoppelung zahlreicher öffentlicher Dienstleistungsbranchen vom Tarifvertragssystem des öffentlichen Dienstes geführt haben.

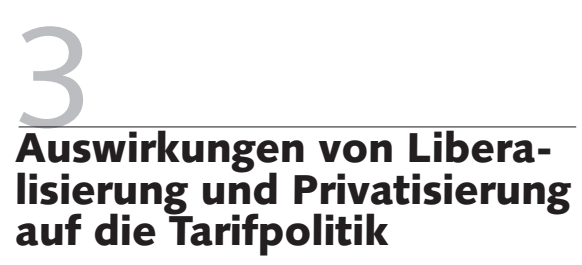

Die Liberalisierung und Privatisierung öffentlicher Dienstleistungen führt zu einer weitreichenden Durchkapitalisierung von gesellschaftlichen Bereichen, die bis dato den Verwertungsmöglichkeiten gewinnorientierten Kapitals entzogen waren. Mit der Liberalisierung werden zunächst ehemals öffentliche Monopole aufgebrochen und neue Märkte geschaffen. Mit den unterschiedlichen Formen der Privatisierung wird in verschiedener Weise auf den neuen Konkurrenzdruck im Rahmen liberalisierter Märkte reagiert (Killian et al. 2006): Die erste Form bildet die formale oder Organisationsprivatisierung, bei der eine ehemals öffentliche Körperschaft in ein Unternehmen mit privater Rechtsform überführt wird, das sich jedoch noch mehrheitlich im öffentlichen Eigentum befindet. Ziel der Organisationsprivatisierung ist vor allem die ökonomische Eigenständigkeit, die dem Management weitgehende Handlungsfreiheit einräumt und vom Einfluss der politischen Institutionen unabhängig macht. Die zweite Form bildet die Aufgabenprivatisierung, bei der bestimmte Tätigkeiten in öffentlichen Unternehmen an private Unternehmen ausgegliedert werden. Schließlich kommt es bei der dritten Form der materiellen oder Vermögensprivatisierung zum tatsächlichen Verkauf öffentlicher Unternehmen an private Investoren.

Unabhängig von der Rechts- und Eigentumsform wird mit der Liberalisierung ein Vermarktlichungsprozess in Gang gesetzt, der das traditionelle Arbeitsregime des öffentlichen Sektors unter einen wettbewerbsbedingten Anpassungsdruck setzt und mit weitreichenden Folgen für die Arbeits- und Beschäftigungsbedingungen sowie die traditionellen Strukturen der Tarifpolitik verbunden ist. So kommt es in der Regel in den von der Liberalisierung betroffenen Branchen zu einer weitgehenden Abkoppelung vom Tarifvertragssystem des öffentlichen Dienstes. An seine Stelle treten neue, vielfach fragmentierte Tarifvertragsstrukturen, die durch einen doppelten Dualismus gekennzeichnet sind (Brandt/ Schulten 2007): Zum einen bildet sich auf sektoraler Ebene ein zweigeteiltes Tarifvertragssystem heraus, mit relativ stabilen Ta- 
rifstrukturen bei den ehemaligen Staatsmonopolisten und den verbliebenen öffentlichen Unternehmen einerseits und eher dezentralisierten Tarifstrukturen auf Seiten der neuen Wettbewerber, die zudem eine deutlich niedrigere Tarifbindung aufweisen. Zum anderen vollzieht sich auf Unternehmensebene eine zunehmende Spaltung zwischen verschiedenen Beschäftigtengruppen, die sich in unterschiedlichen tarifvertraglichen Regelungen für Alt- und Neubeschäftigte und Stamm- und Randbelegschaften niederschlagen. Hinzu kommt eine umfangreiche Ausgliederung von einzelnen Unternehmensbereichen, die oft mit dem Wechsel in einen „billigeren“ Tarifvertrag oder sogar einer vollständigen Tarifflucht einhergeht.

Aufgrund des Fehlens branchenweiter Tarifstandards in den neuen liberalisierten Märkten wird die Konkurrenz über die Arbeitskosten ausgetragen und setzt damit die Beschäftigten unter ernormen Anpassungsdruck. Dies gilt insbesondere für arbeitsintensive Dienstleistungsbereiche wie z. B. die Post, den öffentlichen Personennahverkehr (ÖPNV) oder die Krankenhäuser, wo die Gesamtkosten nach wie vor zu annähernd zwei Dritteln aus Personalkosten bestehen. In einigen dieser Branchen kommt darüber hinaus verschärfend hinzu, dass es sich keineswegs um Wachstumsmärkte handelt, sodass die Konkurrenz die Form eines Verdrängungswettbewerbs annimmt, bei dem am Ende automatisch Arbeitsplätze verloren gehen. Seit Anfang der 1990er Jahre sind in den von Privatisierung und Liberalisierung betroffenen Sektoren mindestens 600.000 Arbeitsplätze abgebaut worden (Brandt/Schulten 2008a, S. 76). Zugleich haben sich die Arbeitsbedingungen für viele Beschäftigte deutlich verschlechtert und es kam zu niedrigeren Löhnen, längeren Arbeitszeiten und einer wachsenden Zahl prekärer Beschäftigungsverhältnisse (Atzmüller/Hermann 2004b).

\section{4 \\ Tarifvertragsstrukturen in liberalisierten Dienstleistungsbranchen}

Der Vergleich von Tarifvertragsstrukturen in den liberalisierten Dienstleistungssektoren zeigt, dass je nach Branche die öffentlichen Unternehmen teilweise tarifpolitisch immer noch direkt an die Tarifverträge des

\begin{tabular}{|c|c|c|}
\hline \multicolumn{3}{|c|}{$\begin{array}{l}\text { Übersicht 1: Tarifstrukturen und Tarifkonkurrenzen in liberalisierten } \\
\text { und privatisierten Branchen }\end{array}$} \\
\hline Branche & $\begin{array}{l}\text { Öffentliche Unternehmen } \\
\text { und ehemalige Staatmonopolisten }\end{array}$ & Private Unternehmen \\
\hline Telekommunikation & $\begin{array}{l}\text { Deutsche Telekom AG: } \\
\text { Haustarifverträge (differenziert nach } \\
\text { Konzernsparten) }\end{array}$ & Haustarifverträge oder tariflos \\
\hline \multirow[t]{2}{*}{ Post/Brief } & $\begin{array}{l}\text { Deutsche Post AG: Haustarifverträge } \\
\text { (differenziert nach Konzernsparten) }\end{array}$ & $\begin{array}{l}\text { Tariflos; rechtlich umstrittene Mindest- } \\
\text { lohntarifverträge mit der "Gewerk- } \\
\text { schaft Neue Brief- und Zustelldienste } \\
\text { (GNBZ)" und dem "Christlichen } \\
\text { Gewerkschaftsbund" }\end{array}$ \\
\hline & \multicolumn{2}{|c|}{$\begin{array}{l}\text { Allgemeinverbindlicher Mindestlohntarifvertrag für Briefdienstleistungen } \\
\text { nach dem Entsendegesetz }\end{array}$} \\
\hline Bahn & $\begin{array}{l}\text { Deutsche Bahn: Haustarifverträge } \\
\text { (differenziert nach Konzernsparten); } \\
\text { Tarifloses Tochterunternehmen } \\
\text { DB Heidekrautbahn }\end{array}$ & $\begin{array}{l}\text { Haustarifverträge, regionale } \\
\text { Verbandstarifverträge oder tariflos }\end{array}$ \\
\hline $\begin{array}{l}\text { Öffentlicher } \\
\text { Personennahverkehr } \\
\text { (ÖPNV) }\end{array}$ & $\begin{array}{l}\text { Spartentarifvertrag TV-Nahverkehr, } \\
\text { Haustarifverträge }\end{array}$ & $\begin{array}{l}\text { Teilweise regionale Verbandstarif- } \\
\text { verträge (Omnibusgewerbe), } \\
\text { Haustarifverträge oder tariflos }\end{array}$ \\
\hline Energieversorgung & $\begin{array}{l}\text { Stadtwerke: Spartentarifvertrag } \\
\text { TV-Versorgung, teils regionale } \\
\text { Flächentarifverträge }\end{array}$ & $\begin{array}{l}\text { Konzerntarifverträge, regionale } \\
\text { Flächentarifverträge }\end{array}$ \\
\hline \multirow[t]{2}{*}{ Entsorgung } & $\begin{array}{l}\text { Tarifvertrag Öffentlicher Dienst } \\
\text { (TV-ÖD) }\end{array}$ & $\begin{array}{l}\text { Branchentarifvertrag für private } \\
\text { Entsorger mit dem Bundesverband } \\
\text { Deutsche Entsorgungswirtschaft (BDE) }\end{array}$ \\
\hline & & $\begin{array}{l}\text { Viele kleine private Unternehmen: } \\
\text { tariflos }\end{array}$ \\
\hline \multirow[t]{2}{*}{ Krankenhäuser } & $\begin{array}{l}\text { TV-ÖD oder Tarifvertrag für den } \\
\text { öffentlichen Dienst der Länder (TV-L); } \\
\text { teilweise Haustarifverträge }\end{array}$ & $\begin{array}{l}\text { Freigemeinnützige Krankenhäuser: } \\
\text { Haustarifverträge oder tariflos } \\
\text { (Sonderregelungen bei den kirchlichen } \\
\text { Häusern) }\end{array}$ \\
\hline & & $\begin{array}{l}\text { Private Krankenhäuser: } \\
\text { Haustarifverträge oder tariflos }\end{array}$ \\
\hline
\end{tabular}

öffentlichen Dienst gebunden sind, zum Teil spezielle Spartentarifverträge abgeschlossen haben oder im Fall der ehemaligen Staatsmonopolisten wie Post oder Bahn Haustarifverträge besitzen, die im Hinblick auf die Lohn- und Arbeitsbedingungen mit denen im öffentlichen Dienst vergleichbar sind. Die neuen privaten Unternehmen sind demgegenüber kaum an Branchentarifverträge gebunden, sondern haben zumeist lediglich Haustarifverträge oder unterliegen überhaupt keiner Tarifbindung (Übersicht 1).

\subsection{TELEKOMMUNIKATION}

In der Telekommunikationsbranche existiert auf Sektorebene eine äußerst fragmentierte Tariflandschaft, die im Wesentlichen aus Haustarifverträgen besteht (Sauerland 2008). Nach der Privatisierung der Telekommunikationssparte der ehemaligen Deutschen Bundespost und der Herausbildung der Deutschen Telekom AG im Jahr 1995 kam es zu einer umfassenden Unternehmensrestrukturierung, in deren Folge im Jahr 2001 eine neues Tarifvertragswerk vereinbart wurde, dass die vorherigen Tarifverträge, die eng an den öffentlichen
Dienst angelehnt waren, ablöste. Parallel hierzu wurde die „Vertöchterungspolitik“ des Telekomkonzerns auch tarifpolitisch nachvollzogen, sodass aus einem ursprünglich einheitlichen Tarifbereich zwölf eigenständige Haustarifverträge für einzelne Tochterunternehmen des Telekomkonzerns entstanden (ebd., S. 102). Während die Altbeschäftigten in der Regel einen tarifpolitischen Bestandsschutz erhielten, wurden die Neueingestellten oft erheblich schlechter gestellt. Zuletzt hat die Deutsche Telekom im Jahr 2007 etwa 55.000 Beschäftigte in drei Servicegesellschaften ausgegliedert. Nach einem elfwöchigen Arbeitskampf wurde zwar eine Absicherung der bestehenden Einkommen und eine mehrjährige Beschäftigungssicherung vereinbart. Zugleich wurden jedoch die Einstiegsentgelte dauerhaft um 6,5\% abgesenkt und die Wochenarbeitszeit wurde von 34 auf 38 Stunden ohne Lohnausgleich verlängert (Schröder 2007).

Seit der Liberalisierung der Telekommunikationsbranche im Jahr 1998 sind zahlreiche neue Telekommunikationsunternehmen auf dem Markt aktiv. In den meisten Fällen handelt es sich um kleinere und mittelständische Unternehmen mit 
lokaler oder regionaler Reichweite, in denen zumeist überhaupt keine Tarifverträge existieren. Bei den neuen landesweit bedeutsamen Mobilfunkunternehmen Vodafone, Arcor und $\mathrm{O} 2$ bestehen Haustarifverträge, die jedoch nicht von der für die Telekom zuständigen Gewerkschaft ver.di, sondern von der IG Metall, von Transnet oder der IG BCE abgeschlossen wurden. Die Gründe hierfür liegen in der Geschichte dieser Unternehmen, die ursprünglich aus dem Metallsektor, der Deutschen Bundesbahn oder dem Chemiesektor stammen. Das bundesweit tätige Mobilfunkunternehmen E-Plus unterliegt hingegen bis heute keiner Tarifbindung.

\subsection{POST}

Ähnlich wie die Deutsche Telekom verfügt auch die Deutsche Post AG über eine Reihe von Haustarifverträgen für die einzelnen Tochterunternehmen innerhalb des Konzerns mit teilweise recht unterschiedlichen Lohn- und Arbeitsbedingungen. Bis Ende 2000 wurden Löhne für die Beschäftigten der ehemaligen Bundespost über den „Tarifvertrag Bundespost" geregelt, der sich an den Lohnsteigerungen des BAT für den öffentlichen Dienst orientierte. Die endgültige Abkopplung von der Lohnentwicklung des öffentlichen Dienstes vollzog sich nach dem Börsengang der Deutschen Post AG im Jahr 2000 (Brandt/Schulten 2007, S. 14). Mit dem Abschluss eines neuen Rahmentarifvertrags für die Arbeiter im Jahr 2001 und die Angestellten im Jahr 2003 wurde innerhalb der Deutschen Post AG eine neue Entgeltstruktur eingeführt. Faktisch wurde dabei eine "Zwei-Klassen-Tarifstruktur“ etabliert, wonach die bis 2001 Eingestellten fortan unter den Tarifvertrag „DP AG Besitzstand“ fielen, während für alle Neueingestellten (mittlerweile etwa ein Viertel aller Tarifbeschäftigten) der „DP AG Tarifvertrag" angewendet wird. Die Unterschiede im Entgeltniveau zwischen beiden Tarifverträgen können bis zu $30 \%$ betragen (Brandt et al. 2007, S. 270).

Mit der sukzessiven Liberalisierung des Briefmarktes seit 1999, die zum 1. Januar 2008 vollständig abgeschlossen wurde, ist eine Vielzahl neuer privater Postunternehmen entstanden, die allesamt keiner Tarifbindung unterliegen. Dies gilt auch für die beiden wichtigsten Konkurrenzunternehmen PIN AG Group und TNT, deren Lohnniveau im Jahr 2006 zwischen $30 \%$ und $60 \%$ unter dem Niveau der Deutschen
Post lag (ebd.). Angesicht dieser zum Teil extrem niedrigen Löhne bei den neuen privaten Postunternehmen, wurde auf Initiative von ver.di und der Deutschen Post im Herbst 2007 ein Mindestlohntarifvertrag vereinbart, der nach dem Entsendegesetz für allgemeinverbindlich erklärt wurde (Brandt/Schulten 2008b). Insbesondere das niederländische Postunternehmen TNT weigert sich jedoch bis heute, diesen Mindestlohn anzuerkennen und hat zunächst mit der "Gewerkschaft Neue Brief- und Zustelldienste (GNBZ) “ und später mit dem "Christlichen Gewerkschaftsbund“ einen eigenen Tarifvertrag mit deutlich niedrigeren Mindestlohnsätzen vereinbart. Gegenwärtig wird vor dem Arbeitsgericht die rechtliche Zulässigkeit des allgemeinverbindlichen Postmindestlohns überprüft.

\subsection{BAHN}

Bis zur Organisationsprivatisierung der Deutschen Bundesbahn im Jahr 1994 wurden in den formell eigenständigen Tarifverhandlungen im Wesentlichen die Tarifergebnisse des öffentlichen Dienstes übernommen (Keller 2007). Mittlerweile existieren innerhalb der Deutschen Bahn AG für die jeweiligen Konzernsparten eigenständige Haustarifverträge. Hinzu kommt eine teilweise berufsgruppenspezifische Zersplitterung der Tariflandschaft, nachdem es 2007 der Gewerkschaft der Lokomotivführer (GDL) erstmals gelungen ist, für die Lokführer eigenständige Tarifbestimmungen $\mathrm{zu}$ vereinbaren (Bispinck/ Dribbusch 2008). Schließlich verfügt die Deutsche Bahn AG mit der DB Heidekraut $\mathrm{GmbH}$ auch über ein nicht tarifgebundenes Tochterunternehmen, in dem Löhne gezahlt werden, die bis zu $20 \%$ unterhalb des ansonsten im Konzern üblichen Niveaus liegen. Gegenwärtig befürchtet die Gewerkschaft Transnet, dass die von der Deutschen Bahn angekündigte Gründung von bis zu 30 Tochtergesellschaften für den Regionalverkehr dem tariflosen Vorbild der Heidekrautbahn folgen könnte.

Gerade im regionalen Schienenverkehr konkurrieren mittlerweile zahlreiche neue Verkehrsunternehmen - darunter auch Tochterunternehmen international operierender Verkehrskonzerne wie Connex, Keolis oder SBB - um öffentliche Aufträge. Sie haben in der Regel Haustarifverträge oder werden durch regionale Verbandstarifverträge erfasst; einige Unternehmen sind auch nicht tarifgebunden. Aufgrund der Überschneidungen zum ÖPNV ist zum Teil neben den Bahngewerkschaften auch ver.di in diesen Bereichen tarifpolitisch tätig. Generell liegt das Tarifniveau der neuen Wettbewerber jedoch zumeist deutlich unterhalb der Deutschen Bahn AG und setzt diese damit unter Druck. Um die Konkurrenz zu begrenzen und die Ausdehnung von Niedriglöhnen einzudämmen, schlägt Transnet vor, für den gesamten Schienenverkehr einen Mindestlohntarifvertrag zu vereinbaren, der ähnlich wie in der Bauindustrie für unterschiedliche Qualifikationsstufen allgemein gültige Mindestlöhne festlegen soll (Kirchner 2008, S. 161f.).

\section{4 ÖFFENTLICHER PERSONEN- NAHVERKEHR (ÖPNV)}

Die öffentlichen Verkehrsbetriebe fallen traditionell in den Geltungsbereich der Tarifverträge für den öffentlichen Dienst. Das vermehrte Auftreten privater Verkehrsunternehmen sowie die veränderte Ausschreibungspraxis der Kommunen hat jedoch die Tariflandschaft im ÖPNV grundlegend verändert (Brandt/Schulten 2008a, S. 82ff.). Mittlerweile existiert in den meisten Bundesländern mit dem Tarifvertrag Nahverkehr (TV-N) ein sogenannter Spartentarifvertrag, der sich vor allem in den Manteltarifbestimmungen immer noch an den öffentlichen Dienst anlehnt, bei der Entgelttabelle das Niveau gegenüber Letzterem jedoch deutlich abgesenkt hat. Die ursprüngliche Idee des Spartentarifvertrags bestand darin, einen neuen Flächentarifvertrag für den ÖPNV zu schaffen, der auch die noch einmal deutlich schlechter vergüteten Bereiche im privaten Verkehrssektor (z. B.im privaten Omnibusgewerbe) einbeziehen sollte. Faktisch existiert heute ein Nebeneinander von TV-N, der die große Mehrheit der öffentlichen Verkehrsbetriebe abdeckt, und verschiedenen regionalen Verbands- und Haustarifverträgen oder auch tariflosen Zuständen in privaten Verkehrsunternehmen. Die hierdurch entstehenden Lohndifferenzen können für dieselbe Tätigkeit bis zu $50 \%$ betragen (ebd.). Eine wichtige stabilisierende Funktion für das Tarifvertragssystem im ÖPNV geht derzeit noch von den in den Anwendungsvereinbarungen zum TV-N ausgehandelten Ausschreibungsquoten aus, die den Anteil der Verkehrsleistungen, die von den Kommunen fremd vergeben werden dürfen, beschränken. 


\subsection{ENERGIEWIRTSCHAFT}

In der Energiewirtschaft ist die Struktur der Tarifverträge äußerst vielschichtig. Mit der Liberalisierung des Strommarktes wurden neben dem Wegfall der Stromaufsicht auch die Gebietsmonopole der großen Stromversorgungsunternehmen rechtlich aufgehoben. Die öffentliche Hand gab Beteiligungen an den großen Stromkonzernen auf. Auf lokaler Ebene verkauften viele Stadtwerke Anteile an die großen Stromkonzerne, von denen seit 2002 nach Fusionswellen nur noch vier Konzerne übrig blieben (Brandt/Schulten 2007). Die Beschäftigten von E.ON, RWE AG und Vattenfall Europe werden über Konzerntarifverträge entlohnt. EnBW AG fällt unter einen regionalen Flächentarifvertrag. Weitere regionale Flächentarifverträge existieren in den Bundesländern für viele Regionalversorger und einige Stadtwerke. Dagegen existiert für die überwiegend im kommunalen Besitz befindlichen Stadtwerke der Tarifvertrag Versorgungsbetriebe (TV-V), der als Spartentarifvertrag des Tarifwerks für den öffentlichen Dienst (BAT/BMTG) im Jahr 2000 entstanden ist. Die materiellen Unterschiede liegen bezogen auf das Jahreseinkommen bei etwa $40 \%$. Diese großen Unterschiede bringen die Gefahr, dass die Tarifregelungen in einen Dumpingkreislauf geraten. Eine größere Tarifkoordinierung und ein mittelfristig anzustrebender Branchentarifvertrag ist aus Sicht von ver.di der einzige Weg, um zukünftiges Tarifdumping und Tarifflucht durch Outsourcing einzudämmen (Bergelin 2008).

\subsection{ENTSORGUNG}

Die Entsorgungswirtschaft weist eine zweigeteilte Tariflandschaft auf (Mendroch 2008). Kommunale Entsorgungsunternehmen fallen zumeist nach wie vor unter den Geltungsbereich des TV-ÖD. Für den privaten Entsorgungssektor besteht ein Branchentarifvertrag zwischen ver.di und dem Bundesverband Deutsche Entsorgungswirtschaft (BDE), der jedoch nur knapp ein Drittel der Beschäftigten erfasst. Mehr als zwei Drittel aller Beschäftigten in privaten Entsorgungsunternehmen ist hingegen nicht tarifgebunden. Der Wettbewerb in der Branche wird vor allem durch die Vergabepraxis der Kommunen angeheizt, die die Unternehmen unter einen erheblichen Kostendruck setzt, den diese direkt an die Beschäftigten weitergeben. Um der damit verbundenen Absenkung von Lohniveaus entgegenzutreten, haben ver.di, der BDE und die Vereinigung der kommunalen Arbeitgeberverbände (VKA) Verhandlungen über die Einführung eines branchenspezifischen Mindestlohns aufgenommen, der nach dem Entsendegesetz allgemeinverbindlich erklärt werden soll. Allerdings wenden sich insbesondere die klein- und mittelständischen privaten Entsorgungsbetriebe mit Vehemenz gegen einen solchen Mindestlohn.

\subsection{KRANKENHÄUSER}

Im Krankenhaussektor erfolgte die Liberalisierung seit den 1990er Jahren insbesondere durch eine grundlegende Reform der Krankenhausfinanzierung. Mit der Einführung von gedeckelten Budgets und Fallpauschalen gerieten die Krankenhäuser immer stärker in einen Kostenwettbewerb, der bei der politisch gewollten Reduzierung der Krankenhauskapazitäten teilweise einen Verdrängungswettbewerb auslöste. Angesicht des hohen Personalkostenanteils von mehr als $60 \%$ wird dieser Wettbewerb vor allem auf dem Rücken der Beschäftigten ausgetragen. Hinzu kommt, dass die über Jahre angestaute Unterfinanzierung öffentlicher Krankenhäuser in eine anhaltende Privatisierungswelle mündete, die zur Herausbildung privater gewinnorientierter Krankenhauskonzerne wie Rhön-Klinikum, Helios oder Asklepios geführt hat.

Die Tarifvertragsstruktur im Krankenhaussektor ist stark zersplittert (Brandt/ Schulten 2008b; Gröschl-Bahr/Stumpfögger 2008). Die öffentlichen Krankenhäuser fallen in den Geltungsbereich der Tarifverträge des öffentlichen Dienstes. Angesichts der schwierigen finanziellen Situation hat eine Reihe öffentlicher Krankenhäuser mittlerweile Notlagentarifverträge abgeschlossen, die ihnen eine zeitweilige Absenkung des Tarifniveaus ermöglichen. Nicht gewinnorientierte Krankenhäuser, die von kirchlichen und anderen Wohlfahrtsverbänden betrieben werden, stellen traditionell einen relativ großen Teil dieses Sektors. Sie haben sich in der Vergangenheit stets eng an den Tarifverträgen des öffentlichen Dienstes orientiert. Mit der Verschärfung des Kostenwettbewerbs ist diese Anbindung in den letzten Jahren jedoch zunehmend brüchig geworden (Kühnlein/Wohlfahrt 2006). Die privaten Krankenhäuser haben in der Regel Haustarifverträge oder sind nicht tarifgebunden, wobei es ver.di in jüngster Zeit gelungen ist, insbesondere bei den großen privaten Krankenhauskonzernen Tarifverträge durchzusetzen, die sich am TV-ÖD orientieren. Insgesamt liegen die Personalkosten in privaten Krankenhäusern im Durchschnitt jedoch um etwa $10 \%$ niediger als in öffentlichen Häusern (Brandt/Schulten 2008b).

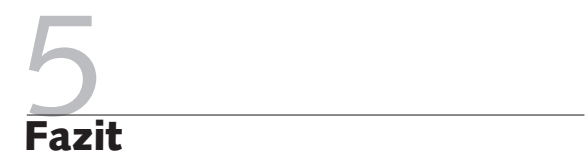

Neben der Dezentralisierung der Tarifpolitik in der Industrie und der geringen Tarifbindung in den privaten Dienstleistungssektoren hat die Liberalisierung und Privatisierung öffentlicher Dienstleistungen als ein dritter zentraler Faktor die Erosion des deutschen Flächentarifvertragssystems befördert. Das ehemals weitreichende Tarifvertragsgefüge des öffentlichen Dienstes bleibt zunehmend auf die Kernbereiche der öffentlichen Verwaltung beschränkt, während durch die Liberalisierung neue Märkte geschaffen werden, die nicht in eine umfassende tarifpolitische Regulierung eingebettet sind. Die Tarifvertragsstrukturen in den liberalisierten Dienstleistungsbranchen sind im Gegenteil hochgradig fragmentiert und deshalb auch nicht in der Lage, den Wettbewerb um Lohn- und Arbeitskosten $\mathrm{zu}$ begrenzen. Im Ergebnis kommt es zu einer umfassenden Re-Kommodifizierung der ökonomischen und sozialen Beziehungen im öffentlichen Sektor (Atzmüller/Hermann 2004a; McDonald/ Ruiter 2006).

Für die Gewerkschaften, deren poltisch-ökonomische Grundaufgabe in der Begrenzung des Wettbewerbs zwischen den Arbeitnehmern liegt, stellt sich vor diesem Hintergrund die Frage, welche politischen Ansatzpunke sich identifizieren lassen, um in den neuen liberalisierten Märkten wieder zu einheitlichen Branchen(mindest)standards zu gelangen. Angesichts der stark fragmentierten Tarifstrukturen ist die wichtigste Voraussetzung hierfür zunächst, dass sich die Gewerkschaften selbst in die Lage versetzen, ihre Tarifpolitik branchenweit zu koordinieren. Dies ist organisationspolitisch alles andere als ein leichtes Unterfangen, zumal in einigen Branchen wie z. B. Telekommunikation, ÖPNV oder der Bahn mehrere Gewerkschaften tätig sind und damit eine gewerkschaftsübergreifende Kooperation nötig ist. 
Ein weiterer Ansatzpunkt, der in einigen Branchen diskutiert und im Falle des Postsektors bereits vereinbart wurde, ist die Festlegung von branchenweiten Mindestlöhnen, die über das Entsendegesetz für alle aus- und inländischen Unternehmen allgemeinverbindlich erklärt werden. Eine solche Strategie findet durchaus auch bei jenen Teilen des Arbeitgeberlagers Unterstützung, die an der Verhinderung von Schmutzkonkurrenz und in diesem Sinne fairen Wettbewerbsbedingungen interessiert sind. Allerdings haben die Arbeitgeber bei diesem Ansatz eine starke Vetomacht, die es ihnen jederzeit erlaubt, ihre Zustimmung für einen Mindestlohntarifvertrag zu versagen. Deshalb müsste eine solche Strategie durch die Einführung eines allgemeinen, branchenübergreifenden gesetzlichen Mindestlohns zusätzlich gestärkt werden (Bispinck/Schulten 2008).

Mit dem Mindestlohn allein lässt sich jedoch nur ein Teil der Lohn- und Arbeitskostenkonkurrenz begrenzen. Perspektivisch stellt sich deshalb die Frage, wie es gelingen kann, die heterogenen Tarifstrukturen in den liberalisierten und privatisierten Branchen wieder zu vereinheitlichen und neue branchenweite Flächentarifverträge abzuschließen. In den 1990er Jahren gab es hierzu innerhalb der Gewerkschaften eine umfassende Debatte über die Einrichtung von Spartentarifverträgen, deren Grundidee darin bestand, die Anbindung an den Tarifvertrag und die Tarifrunden im öffentlichen Dienst aufrechtzuerhalten und gleichzeitig neue private Unternehmen in die branchenspezifischen Regelungen der Spartentarifverträge einzubinden. Der Spartentarifvertrag TV-Nahverkehr liegt daher im Entgeltniveau unterhalb des TV-ÖD, aber oberhalb existierender Tarifverträge mit privaten Verkehrsunternehmen. Die existierenden Spartentarifverträge haben jedoch nicht zur Etablierung neuer branchenweiter Flächentarifverträge geführt. Ursprünglich sollte der Abschluss eines Spartentarifvertrags im ÖPNV an die Durchsetzung einer Tariftreueregelung gekoppelt werden, wonach der Staat sich verpflichtet, nur denjenigen Verkehrsunternehmen einen Auftrag zu erteilen, die den Spartentarifvertrag anwenden. In der Praxis sind solche Tariftreueregelungen zum ÖPNV jedoch nur in einigen wenigen Bundesländern umgesetzt worden, während ein bundesweites Tariftreuegesetz am politischen Widerstand der Unionsparteien scheiterte (Schulten/Pawicki 2008).

Der Europäische Gerichtshof (EuGH) hat in einem Grundsatzurteil vom April 2008 die Tariftreueregelungen in Deutschland als Verstoß gegen die europäische Dienstleistungsfreiheit gewertet und damit für unzulässig erklärt. Damit ergibt sich eine groteske Situation: Dadurch, dass der Staat als öffentlicher Auftraggeber verpflichtet ist, das „wirtschaftlichste Angebot" anzunehmen, ist er im Prinzip gezwungen, Unternehmen zu bevorzugen, die aufgrund fehlender Tarifbindung günstigere Angebote machen. Damit würde der Staat selbst zu einer weiteren Erosion des Tarifvertragssystems beitragen. Gesetzliche Tariftreuereglungen bleiben deshalb eine zentrale Voraussetzung zur Schaffung neuer branchenweiter Flächentarifverträge in den liberalisierten Branchen und müssen entsprechend europarechtlich abgesichert werden.
Ohne Tariftreueregelungen bleiben nur die Allgemeinverbindlichkeit von Branchentarifverträgen sowie die Übernahme des gesamten Tarifvertrags in das Entsendegesetz, um nach dem Wegfall der Freizügigkeitsgrenzen in Deutschland ab dem Jahr 2011 auch ausländische Unternehmen zur Einhaltung der gleichen Standards zu zwingen. Allerdings ist das Instrument der Allgemeinverbindlichkeit in Deutschland im Gegensatz zu vielen anderen europäischen Ländern äußerst restriktiven Regeln unterworfen und gibt den Arbeitgebern eine starke Blockademacht, die in der Praxis dazu geführt hat, dass der Anteil allgemeinverbindlicher Tarifverträge seit den 1990er Jahren immer mehr zurückgegangen ist. Eine stärkere Anwendung dieses Instruments hat deshalb eine grundlegende Reform zur Vereinfachung und Erleichterung von Allgemeinverbindlichkeitserklärungen zur Voraussetzung (Zachert 2004).

Die Erosion des Flächentarifvertrags im Zuge der Liberalisierung und Privatisierung öffentlicher Dienstleistungen macht die politische Verantwortung für diesen Prozess besonders deutlich. Im Gegensatz $\mathrm{zu}$ den meisten anderen westeuropäischen Staaten verfügt Deutschland kaum über Institutionen, die das Tarifvertragssystem politisch stützen (Schulten 2004). Der in der Öffentlichkeit weit fortgeschrittene Legitimationsverlust von Liberalisierung und Privatisierung, der nicht zuletzt auch durch die negativen Konsequenzen für die Beschäftigten begründet ist, könnte einen Ansatzpunkt bieten, um auch hierzulande über die politische Re-Stabilisierung des Flächentarifvertrages nachzudenken.

\section{LITERATUR}

Artus, I. (2001): Krise des deutschen Tarifsystems. Die Erosion des Flächentarifvertrags in Ost und West, Wiesbaden Atzmüller, R./Hermann, C. (2004a): Veränderung öffentlicher Beschäftigung im Prozess der Liberalisierung und Privatisierung, in: Österreichische Zeitschrift für Soziologie (ÖZS) 4, S. 30-48

Atzmüller, R./Hermann, C. (2004b): Liberalisierung öffentlicher Dienstleistungen in der EU und Österreich. Auswirkungen auf Beschäftigung, Arbeitsbedingungen und Arbeitsbeziehungen, in: Kammer für Arbeiter und Angestellte Wien: Zur Zukunft öffentlicher Dienstleistungen 2, Wien
Bergelin, S. (2008): Energiewirtschaft, in: Brandt, T./Schulten, T./Sterkel, G./Wiedemuth, J. (Hrsg.): Europa im Ausverkauf. Liberalisierung und Privatisierung öffentlicher Dienstleistungen und ihre Folgen für die Tarifpolitik, Hamburg, S. 121-130

Bispinck, R. (2006a): Abschied vom Flächentarifvertrag? Der Umbruch in der deutschen Tariflandschaft, in: WSI-Tarifhandbuch 2006, Frankfurt/Main, S. 41-66

Bispinck, R. (2006b): Mehr als nur ein Streit um 18 Minuten - Die Tarifauseinandersetzung im öffentlichen Dienst 2006, in: WSI-Mitteilungen 7, S. 374-388 
Bispinck, R. (Hrsg.) (2007): Wohin treibt das Tarifsystem?, Hamburg. Bispinck, R./Dribbusch, H. (2008): Tarifkonkurrenz der Gewerkschaften zwischen Über- und Unterbietung, in: Sozialer Fortschritt 6, S. 153-163 Bispinck, R./Schulten, T. (2003): Verbetrieblichung der Tarifpolitik? Aktuelle Tendenzen und Einschätzungen aus Sicht von Betriebs- und Personalräten, in: WSI Mitteilungen 3, S. 157-166

Bispinck, R./Schulten, T. (2008): Aktuelle Mindestlohndebatte: Branchenlösungen oder gesetzlicher Mindestlohn? in: WSI-Mitteilungen 3, S. $151-158$

Bordogna, L. (2007): Industrial relations in the public sector, Dublin: European Foundation for the Improvement of Living and Working conditions, Eironline, Comparative Studies: www.eurofound.europa.eu/eiro/ studies/tn0611028s/tn0611028s.html

Brandt, T./Drews, K./Schulten, T. (2007): Liberalisierung des deutschen Postsektors - Auswirkungen auf Beschäftigung und Tarifpolitik, in: WSIMitteilungen 5, S. 266-273

Brandt, T./Schulten, T. (2007): Liberalisation and Privatisation of Public Services and the Impact on Labour Relations: A Comparative View from six Countries at the Postal, Hospital, Local Public Transport and Electricity Sector, Report for the EU-Project "Privatisation of Public Services and the Impact on Quality, Employment and Productivity (PIQUE)". (www. pique.at/reports/pubs/PIQUE_028478_Del8.pdf)

Brandt, T./Schulten, T. (2008a): Auswirkungen von Privatisierung und Liberalisierung auf die Tarifpolitik in Deutschland. Ein vergleichender Überblick, in: Brandt, T./Schulten, T./Sterkel, G./Wiedemuth, J. (Hrsg.) (2008): Europa im Ausverkauf. Liberalisierung und Privatisierung öffentlicher Dienstleistungen und ihre Folgen für die Tarifpolitik, Hamburg, S. 68-259 Brandt, T./Schulten, T. (2008b): Privatisation and liberalisation of public services in Germany: the postal and hospital sector, in: Keune, M./Leschke, J./Watt, A. (Hrsg.): Privatisation and Marketisation of Services Social and Economic Impacts on Employment, Labour Markets and Trade Unions, Brussels: ETUI-REHS, im Erscheinen

Dribbusch, H./Schulten, T. (2007): The End of an Era: Structural Changes in German Public Sector Collective Bargaining, in: Leisink, P./Steijn, B./

Veersma, U. (2007): Industrial Relations in the New Europe, Cheltenham, S. $155-176$

Ellguth, P./Kohaut, S. (2008): Tarifbindung und betriebliche Interessenvertretung: Aktuelle Ergebnisse aus dem IAB-Betriebspanel 2007, in: WSIMitteilungen 9, S. 515-519

Gröschl-Bahr, G./Stumpfögger, N. (2008): Krankenhäuser, in: Brandt, T./ Schulten, T./Sterkel, G. /Wiedemuth, J. (Hrsg.): Europa im Ausverkauf. Liberalisierung und Privatisierung öffentlicher Dienstleistungen und ihre Folgen für die Tarifpolitik, Hamburg, S. 165-180

Keller, B. (1993): Arbeitspolitik des öffentlichen Sektors, Baden-Baden

Keller, B. (2007): Wandel der Arbeitsbeziehungen im öffentlichen Dienst: Entwicklung und Perspektiven, in: Die Verwaltung 2, S. 173-202
Killian, W./Richter, P./Trapp, J. H. (2006): Ausgliederung und Privatisierung in den Kommunen. Empirische Befunde zur Struktur kommunaler Aufgabenwahrnehmung, Berlin Kirchner, A. (2008). Die Bahn, in: Brandt, T./Schulten, T./Sterkel, G. /Wiedemuth, J. (Hrsg.): Europa im Ausverkauf. Liberalisierung und Privatisierung öffentlicher Dienstleistungen und ihre Folgen für die Tarifpolitik, Hamburg, S. 153-164

Kohaut, S./Schnabel, C. (2003): Zur Erosion des Flächentarifvertrags. Ausmaß, Einflussfaktoren und Gegenmaßnahme, in: Industrielle Beziehungen 2, S. 193-219

Kohaut, S./Schnabel, C. (2007): Tarifliche Öffnungsklauseln. Verbreitung, Inanspruchnahme und Bedeutung, in: Sozialer Fortschritt 2, S. 33-40 Kühnlein, G./Wohlfahrt, N. (2006): Soziale Träger auf Niedriglohnkurs? Zur aktuellen Entwicklung der Arbeits- und Beschäftigungsbedingungen im Sozialsektor, in: WSI-Mitteilungen 7, S. 389-395

McDonald, D. A./Ruiter, G. (2006): Rethinking Privatisation. Towards a Critical Theoretical Perspective, in: Chavez, D. (Hrsg.): Beyond the Market: The Future of Public Services, Trans National Institute (TNI) Public Services Yearbook 2005/6, S. 10-20

Mendroch, E. (2008): Abfallwirtschaft, in: Brandt, T./Schulten, T./Sterkel, G./Wiedemuth, J. (Hrsg.): Europa im Ausverkauf. Liberalisierung und Privatisierung öffentlicher Dienstleistungen und ihre Folgen für die Tarifpolitik, Hamburg, S. 131-138

Oppholzer, A./Zachert, U. (Hrsg.) (2000): Krise und Zukunft des Flächentarifvertrages, Baden-Baden

Rehder, B. (2003): Betriebliche Bündnisse für Arbeit in Deutschland. Mitbestimmung und Flächentarif im Wandel. Schriftenreihe des Max-PlanckInstituts für Gesellschaftsforschung Band 48, Frankfurt/New York

Sauerland, F. (2008): Telekommunikation, in: Brandt, T./Schulten, T./ Sterkel, G./Wiedemuth, J. (Hrsg.): Europa im Ausverkauf. Liberalisierung und Privatisierung öffentlicher Dienstleistungen und ihre Folgen für die Tarifpolitik, Hamburg, S. 92-107

Schnabel, C. (2007): Arbeitnehmervertretungen im öffentlichen Sektor: Bedeutung und (potenzielle) Effizienzwirkungen, Diskussionspapier 52 des Lehrstuhls für Arbeitsmarkt- und Regionalpolitik an der FriedrichAlexander-Universität in Erlangen-Nürnberg

Schröder, L. (2007): Der Tarifkonflikt bei der Deutschen Telekom AG, in: WSI-Mitteilungen 9, S. 515-518

Schulten, T. (2004): (Flächen-)Tarifvertragssysteme in Europa, in: WSI (Hrsg.): WSI-Handbuch 2004, Frankfurt a. M., S. 43-64

Schulten, T./Pawicki, M. (2008): Tariftreueregelungen in Deutschland Ein aktueller Überblick, in: WSI-Mitteilungen 4, S. 184-190

Zachert, U. (2004): Allgemeinverbindlicherklärung, in: Peter, G./Kempen, O. E./Zachert, U.: Die Sicherung tariflicher Mindeststandards, BadenBaden, S. 11-32 